\author{
Military Technical College \\ Kobry El-Kobbah, \\ Cairo, Egypt.
}

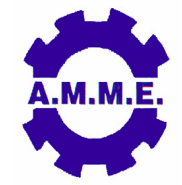

\title{
AN EXPERIMENTAL STUDY ON TURBULENT EMULSION (OIL-IN-WATER) FLUID FLOW THROUGH CURVED DIFFUSER
}

\author{
W. A. El-Askary*, K. A. Ibrahim**, E. Wahba** and H. A. Omara**
}

\begin{abstract}
The paper presents a comprehensive experimental study on emulsion (oil-in-water) fluid flow through curved diffusers. The experimental setup has been designed and constructed in the fluid mechanics laboratory of the faculty of engineering, Menoufiya University to perform the measurements which have been carried out on five models of curved diffusers. The measurements of pressure distributions along the outer and inner walls of the curved diffuser have been performed for different area ratios, different curvature ratios (ratio of centerline arc radius to inlet width), different inflow Reynolds numbers and different emulsion holdup (ratio of oil volume to emulsion volume), while the oil concentration has been varied from $0 \%$ to $20 \%$ by volume. The experimental work has been carried out using two sets of oil-in-water emulsions; the first is stable oil-in-water (o/w) emulsion using Sodium Dodecyl Sulfate (SDS) emulsifier and the second is unstable o/w emulsion at different holdup values. The energyloss coefficients for each model are based on detailed measurements of the wall pressure distributions along walls of the curved-diffuser models including long upstream and downstream tangents. The energy-loss coefficient data have been plotted versus of Reynolds number at different concentrations.
\end{abstract}

New results on energy-loss coefficient during flow of unstable and stable oil-in-water emulsions through curved diffusers have been reported in the present paper. The diffuser energy-loss coefficient is strongly affected by the geometrical parameters of diffuser, Reynolds number and emulsion holdup. Generally for the flow in curved diffusers, the resistance coefficients for stable and unstable (oil-in-water) emulsions are higher than that of pure-water flow. It is also noticed that the unstable o/w emulsion exhibits lower values in loss coefficient compared with that given for stable o/w emulsion.

General correlation of the loss coefficient (not previously considered) for the present cases exploring the ranges of geometrical parameters, inflow condition and Reynolds number conditions is extracted from the present experimental study.

\section{KEY WORDS}

Holdup, Emulsion flow, Stable, Unstable, Energy losses, Curved diffusers models

Assoc. Professor; Mechanical Power Engineering Dept., Faculty of Engineering, Menoufiya University (Corresponding author), Tel.: +2-01005255817, +2-048-3486965; Fax: +2-048-2235695. Email address: wageeh elaskary@yahoo.com.

** Mechanical Power Engineering Dept., Faculty of Engineering, Menoufiya University 


\section{NOMENCLATURE}

\section{Symbols}
A Cross-sectional area, $m^{2}$
$B \quad$ Height of the curved diffuser, $m$
$C_{d} \quad$ Discharge coefficient of the orifice flow meter
$C_{p} \quad$ Static pressure recovery coefficient
$C_{p}(I) \quad$ Ideal Static pressure recovery coefficient
$C_{p_{i}} \quad$ Static pressure recovery coefficient on the inner wall
$C_{p_{o}} \quad$ Static pressure recovery coefficient on the outer wall
$D_{H} \quad$ Hydraulic diameter, $m$
$K_{d} \quad$ Curved diffuser energy loss coefficient
$p \quad$ Pressure, $\mathrm{N} / \mathrm{m}^{2}$
$P_{\text {exit }} \quad$ Curved diffuser exit Pressure, $\mathrm{N} / \mathrm{m}^{2}$
$P_{\text {inlet }} \quad$ Curved diffuser inlet Pressure, $\mathrm{N} / \mathrm{m}^{2}$
$Q \quad$ Fluid Flow Rate, $\mathrm{m}^{3} / \mathrm{s}$
$R_{c} \quad$ Curved diffuser centre-line arc radius, $m$
$\mathrm{Re} \quad$ REYNOLDS number
$U_{\text {ref }} \quad$ Mean-streamwise velocity at the upstream reference location, $\mathrm{m} / \mathrm{s}$
$V_{E} \quad$ Emulsion volume, $\mathrm{m}^{3}$
$V_{\text {exit }} \quad$ Diffuser exit flow velocity, $\mathrm{m} / \mathrm{s}$
$V_{\text {inlet }} \quad$ Diffuser inlet flow velocity, $\mathrm{m} / \mathrm{s}$
$V_{o} \quad$ Oil volume, $\mathrm{m}^{3}$
$V_{w} \quad$ Water volume, $\mathrm{m}^{3}$
$W \quad$ Curved diffuser inlet width, $m$
$W_{\text {exit }} \quad$ Curved diffuser exit width, $m$
$S \quad$ dimensional distance along diffuser centerline, measured from the reference location, $m$
$S^{*} \quad$ Non-dimensional streamwise distance, $(=S / W)$

\section{Greek symbols}

$\Phi \quad$ Holdup (Ratio of oil volume to emulsion volume)

$\mu \quad$ Dynamic viscosity, $\mathrm{cP}$

$\mu_{E} / \mu_{w} \quad$ Emulsion/Water viscosity.

$v \quad$ Kinematic viscosity, centi Stoke.

$\rho_{E} \quad$ Mixture density, $\mathrm{Kg} / \mathrm{m}^{3}$

$\rho_{m} \quad$ Mercury density, $\mathrm{Kg} / \mathrm{m}^{3}$

$\rho_{o} \quad$ Oil density, $\mathrm{Kg} / \mathrm{m}^{3}$ 
$\rho_{w} \quad$ Water density, $\mathrm{Kg} / \mathrm{m}^{3}$

\title{
Subscripts
}

$\begin{array}{ll}d & \text { Diffuser condition. } \\ E & \text { Emulsion } \\ \text { exit } & \text { Exit } \\ \mathrm{I} & \text { Ideal } \\ i & \text { Inner wall (Convex wall) } \\ 0 & \text { Outer wall (Concave wall) } \\ \mathrm{O} & \text { Oil } \\ \mathrm{p} & \text { Pressure recovery } \\ r e f & \text { Reference value } \\ \mathrm{W} & \text { Water } \\ x & \text { Location value } \\ \infty & \text { Free-stream value }\end{array}$

\section{List of Abbreviations}

AR Area-Ratio of the curved diffuser $=A_{\text {exit }} / A_{\text {inlet }}$

$\mathrm{CR}$ Curvature-Ratio of the curved diffuser $=R_{c} / W$

O/W Oil-in-water emulsion

SDS Sodium dodeycl sulfate

W/O Water-in-oil emulsion

WP Wetted perimeter, $\mathrm{m}$

\section{List of Chemical Symbols}

\author{
$\mathrm{CCL}_{4} \quad$ Carbon Tetra Chloride \\ $\mathrm{CH}_{3}\left(\mathrm{CH}_{2}\right)_{11} \mathrm{OSO}_{3} \mathrm{Na}$ Sodium Dodeycl Sulfate
}

\section{INTRODUCTION}

The emulsion is a mixture of two immiscible liquids, one of which is dispersed in the form of small droplets throughout the other. The dispersed liquid is known as the internal or discontinuous phase whereas the dispersion medium is known as the external or continuous phase. As an important application, the two-phase (oil/water) emulsion flow is used for extraction of the petroleum oil from under ground by injecting water. Emulsions are also used in many other industries, such as pharmaceutical, agricultural and food industries. Curved diffusers are necessary in fluid flow to decelerate and turn the fluid simultaneously. Curved Diffusers are used in vaned diffusers of centrifugal pump passages, in steam turbine and in the interconnected ducting between the components of gas turbine. Literature review on single phase flow through curved diffuser indicates that the overall pressure recovery coefficient of the curved diffuser increases by increasing the inflow Reynolds number, by increasing the turbulence intensity and by increasing area 
ratio. Curved diffuser flow constitutes the most complex type of flows because they are rotational and three dimensional in shape for small aspect ratio. Consequently, curved diffuser flows are difficult to investigate both experimentally and numerically. Curved diffuser of varying lengths and aspect ratios has been employed in the past to study the streamline curvature effects and secondary motions in single phase flows. One of the main features of flow in curved diffuser is the presence of centrifugal force (because of the curvature) which gives rise to the radial pressure gradient (normal to the streamline direction) which varies inversely with the radius of curvature of the streamline. Consequently the radial pressure drives secondary motion, creating secondary cells, which are strongly affected by the radius of curvature.

Review of literatures indicated basically two types of experiments on single phase flow. In the first one, developing flow in curved diffusers of large aspect ratio has been measured to study the effects of convex or concave curvature on a nominally two-dimensional turbulent boundary layer. The aspect ratio of curved diffusers is defined as the ratio of its cross-section height to the inlet width. The second type of experiments has been conducted mostly in curved diffuser with short or long straight sections upstream of the curved diffuser to study the evaluation of the secondary motion of developing and fully developed flows.

Pal and Rhodes [1] investigated experimentally the laminar and turbulent flow behaviors of stable oil-in-water $(\mathrm{o} / \mathrm{w})$ emulsions in horizontal pipeline. They noticed that; up to a dispersed phase concentration of $55.14 \%$ by volume, emulsions are Newtonian. For concentration of $65.15 \%$ o/w emulsions are non-Newtonian powerlaw fluids. It was concluded that friction factor for stable o/w emulsions investigated follows the usual equations of single-phase Newtonian and non-Newtonian fluids with averaged properties. It was also pointed out that for dispersed phase concentration $>50 \%$ stable $\mathrm{o} / \mathrm{w}$ emulsions exhibit drag reduction in turbulent flow, i.e.; the experimental friction factors fall somewhat below the single - phase equation.

An experimental study concerning the laminar and turbulent flow behaviors of unstable and stable water-in-oil (w/o) and oil-in-water (o/w) emulsions was performed by Pal [2]. The results showed that; the unstable o/w emulsions exhibit drag reduction behavior in the turbulent regime depending upon the nature of the oil and the holdup of the dispersed phase. The unstable w/o emulsions exhibit much stronger drag reduction activity than the unstable o/w emulsions. The stable emulsions exhibit relatively little drag reduction; therefore the pipeline flow behavior of stable o/w and w/o emulsions could be predicted reasonably well using the usual equations of single-phase flow.

Hwang and $\mathrm{Pal}$ [3] measured the flow of two-phase oil/water mixtures through sudden expansions and contractions experimentally. The energy losses were determined from the measured pressure profiles upstream and downstream. The loss coefficients for the emulsions were found to be independent on the type of emulsion. Also the results indicated that the emulsions are of oil-in-water type up to $64 \%$ (oil volume) and above this ratio the emulsions are water-in-oil type. 
Turian et al. [4] found that the resistance coefficients for non-Newtonian suspension flow through bends of various angles and radii of curvature and through valves decrease with increasing Reynolds number in laminar flow. However, constant asymptotic values of resistance are approached in fully developed turbulent flow.

Pal and Hwang [5] presented an experimental study concerning the loss coefficients for flow of surfactant-stabilized emulsions through pipe components (valve, expansion and contraction). They showed that emulsions are Newtonian only at low to moderate concentrations of dispersed phase (oil concentration up to $46.9 \%$ ). Also, they concluded that the frictional losses can be successfully correlated as loss coefficient versus Reynolds Number.

Khalil et al. [6] performed an experimental study for flow of water with surfactant additives through sudden enlargements, sudden contractions and short bends. The results showed that the reduction in secondary losses increases by increasing of surfactant concentration and Reynolds number and/or decreasing pipe diameter.

Langevin et al. [7] found that dispersing heavy oil or bitumen in water is a very efficient way to reduce the viscosity of the fluid by more than 2 orders of magnitude. Thus, formation of oil-in-water emulsions can be used to reduce the viscosity in pipelines and hence reduction of energy losses and the required power to transmit the oil in pipelines.

Ismail [8] studied theoretically the drag reduction behavior of the unstable w/o emulsions in turbulent flow in a horizontal pipeline. The drag reduction is considered to be due to two reasons, the damping of turbulence due to the presence of dispersed phase, and due to breakage and coalescence processes. Thus, two correlations were obtained to describe the function in saucer mean diameter, holdup and diameter. The correlations were found to fit in good agreement with the experimental results of $\mathrm{Pal}$ [2].

A study of oil-in-water emulsion flow through pipeline using image analysis technique was performed by Khalil et al. [9]. The emulsion was prepared by adding oil to tap water and the results indicated that: Increasing emulsion holdup (ratio of oil volume to mixture volume) causes increasing in emulsion viscosity, increasing oil droplet diameter and decreasing discharge coefficient and also increasing energy-losses coefficient. Energy-loss coefficients of $90^{\circ}$ bend, fully opened gate valve, sudden enlargement and sudden contraction were arranged according to the big value respectively. The resistance coefficient of pipe fittings for emulsion flow is lower than that for water flow. It was concluded that; for holdup lower than $50 \%$ the o/w emulsion flow is Newtonian flow and for holdup higher than $50 \%$ the o/w emulsion flow is non-Newtonian flow.

The pressure drop in sudden contractions was measured for Newtonian and nonNewtonian fluid under different flow Reynolds number by Fester et al. [10]. The experimental study indicated that: Contraction Loss coefficient $\left(K_{c o n}\right)$ decreases with increasing contraction ratio $\left(D_{d} / D_{u}=\right.$ Ratio of downstream pipe diameter to upstream pipe diameter). The study showed that increasing flow Reynolds number decreases contraction loss coefficient. 
The turbulent flow inside a combined bend-diffuser configuration with a rectangular cross section was experimentally and numerically studied by El-Askary and Nasr [11]. The experimental study included the outer and inner-wall-pressure measurements and the overall system/diffuser loss determination. The results show that there is an optimum diffuser angle which depends on the inflow Reynolds number and produces the minimum pressure loss and hence good performance of such complex geometry is obtained.

The pressure drop of oil-water flow through sudden contraction and expansion in a horizontal pipe was measured by Balakhrisna et al. [12]. The abrupt change in area during oil-water flow through pipe strongly affects on the phase distribution. The loss coefficient is independent on the flow patterns. Contraction and expansion coefficients are found to be lower for oil-in-water flow in comparison to pure water flow though the same test rigs.

Hammoud et al. [13] studied the effect of oil-in-water concentration on the performance of a centrifugal pump. They concluded that the head and efficiency decrease by increasing the oil concentration. Increasing in oil concentration leads also to increasing the power consumption.

Khalil et al. [14] measured the energy Losses of oil-in-water emulsions flow through pipe fittings using image processing. They found that the energy loss coefficient increases as the holdup increases and the flow rate decreases; also they showed that the energy loss coefficient is found to be inversely proportional to the generalized flow Reynolds number.

The main objective of the present work is to gain insight into the curved diffuser performance with emulsion (oil-in-water) flow. The effects of the inflow Reynolds number and the geometrical parameters of the curved diffuser such as the curvature ratio and area ratio are considered. The oil concentration in water (holdup) is also included in order to extract general conclusions. First of all, outer and inner wall static pressure distributions are experimentally measured in flow of stable and unstable oil-in-water emulsions through curved diffusers at different values of holdup and flow Reynolds numbers. Second, the energy losses of stable and unstable oil-inwater emulsions flow through curved diffusers at different values of holdup and Reynolds numbers are also extracted.

\section{ANALYTICAL STUDY}

Emulsions can generally be treated as pseudo-homogeneous fluids with averaged properties as the dispersed droplets of emulsions are small and are well distributed. Consequently, one can apply the single-phase flow equation to correlate the pressure loss data for emulsion flow through diffusers. It should be noted that emulsions are Newtonian only at low to moderate concentrations of dispersed phase (oil concentration up to 46.9\%), see Pal and Hwang [5].

Bernoulli's equation is valid along any streamline in any frictionless flow, and it can be modified to include the energy losses due to the presence of curved diffuser wall friction and the superimposed vortices produced by curvature or recirculation. Thus, 
for the $90^{\circ}$ curved diffuser, the modified Bernoulli's equation applied between the inlet and exit of the diffuser is written as:

$$
\begin{aligned}
& h_{\text {inlet }}+\frac{V_{\text {inlet }}^{2}}{2 g}=h_{\text {exit }}+\frac{V_{\text {exit }}^{2}}{2 g}+h_{\text {loss }} \\
& h_{\text {loss }}=\left(h_{\text {inlet }}-h_{\text {exit }}\right)+\frac{\left(V_{\text {inlet }}^{2}-V_{\text {inlet }}^{2} / A R^{2}\right)}{2 g} \\
& h_{\text {loss }}=\frac{\left(P_{\text {inlet }}-P_{\text {exit }}\right)}{\rho_{E} g}+\frac{V_{\text {inlet }}^{2}\left(1-1 / A R^{2}\right)}{2 g}
\end{aligned}
$$

where $h_{\text {inlet }}$ and $h_{\text {exit }}$ are the pressure heads at inlet and exit, respectively. $V_{\text {inlet }}$ and $V_{\text {exit }}$ are the time-averaged mean velocities at inlet and exit sections, respectively and $\rho_{E}$ is the mixture (emulsion) density. The pressure coefficient $\left(C_{p}\right)$ is defined as:

$$
C_{p}=\frac{\left(P_{x}-P_{r e f}\right)}{0.5 \rho_{E} U_{r e f}^{2}}
$$

Where, $P_{x}$ is the local pressure measured at the wall and $P_{r e f}$ is the reference pressure and $U_{\text {ref }}=V_{\text {inlet }}$ is the reference mean velocity. The reference location is taken at a distance $20 \mathrm{~mm}$ upstream of the curved diffuser entrance, where the upstream effect of the diffuser can be omitted.

The energy loss coefficient $\left(K_{d}\right)$ can then be determined from the following equation:

$$
K_{d}=\left(C_{p(\text { inlet })}-C_{p(\text { exit })}+1-\frac{1}{A R^{2}}\right)
$$

From which the loss head $\left(h_{\text {loss }}\right)$ can be computed as:

$$
h_{\text {loss }}=K_{d} \frac{U_{r e f}^{2}}{2 g}
$$

where $C_{p(\text { inlet })}$ and $C_{p(e x i t)}$ are the pressure coefficients at inlet and exit of the diffuser, respectively and $A R=A_{\text {exit }} / E_{\text {inlet }}$ is the diffuser area ratio. The area ratios for the considered geometries are: $A R=2$ for models $1,2,3$ and 4 , and $A R=1.5$ for model 5 , while model 6 constitutes a smooth-curved duct of $A R=1$, see Table 1 .

Table 1. Geometrical parameter of the curved-diffuser models.

\begin{tabular}{|c|c|c|c|c|c|c|c|c|}
\hline Model & $R_{c}(\mathrm{~cm})$ & $W(\mathrm{~cm})$ & $W_{\text {exit }}(\mathrm{cm})$ & $B(\mathrm{~cm})$ & $A_{\text {inlet }}\left(\mathrm{cm}^{2}\right)$ & $A_{\text {exit }}\left(\mathrm{cm}^{2}\right)$ & $C R$ & $A R$ \\
\hline$(1)$ & 25 & 2 & 4 & 8 & 16 & 32 & 12.5 & 2 \\
\hline$(2) /(4)$ & 15 & 2 & 4 & 8 & 16 & 32 & 7.5 & 2 \\
\hline$(3)$ & 10 & 2 & 4 & 8 & 16 & 32 & 5 & 2 \\
\hline$(5)$ & 15 & 2 & 3 & 8 & 16 & 24 & 7.5 & 1.5 \\
\hline$(6)$ & 15 & 2 & 2 & 8 & 16 & 16 & 7.5 & 1 \\
\hline
\end{tabular}




\section{EXPERIMENTAL SETUP AND PROCEDURE}

\section{Apparatus}

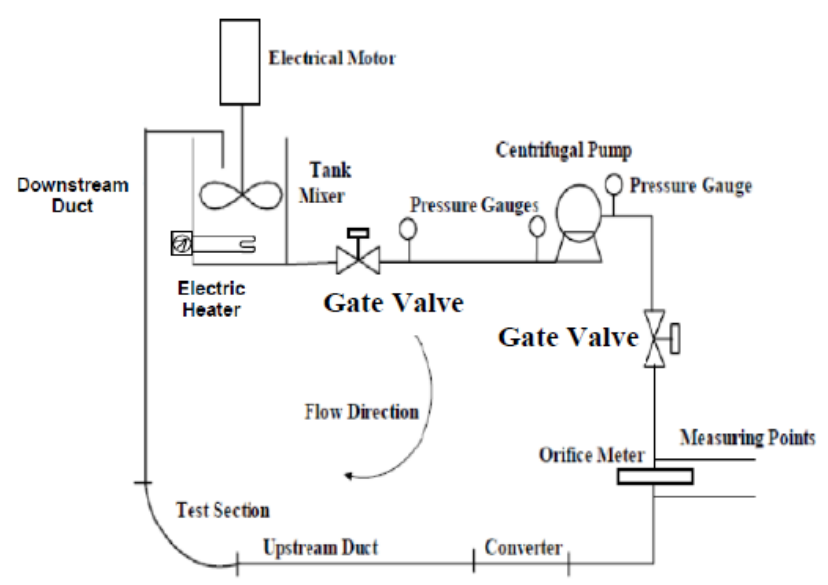

(a)

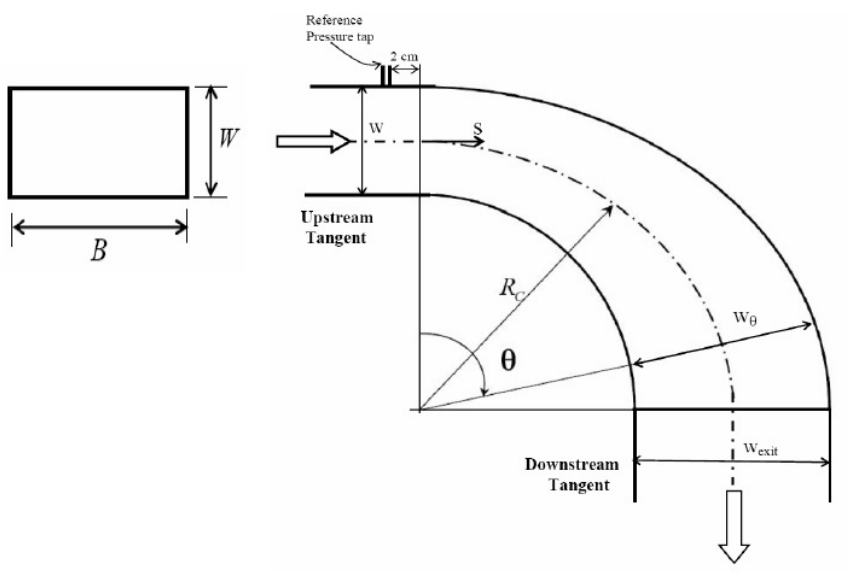

(b)

Fig. 1. Schematic diagram of the experimental setup (a) and test section of the curved diffuser (b) (not to scale).

Figure 1a shows the test rig that is designed to investigate the $\mathrm{o} / \mathrm{w}$ emulsion flow through curved diffusers of constant height $(B)$ and inlet width $(W)$ of $80 \mathrm{~mm}$ and $20 \mathrm{~mm}$, respectively, but different center-line arc radius $\left(R_{c}\right)$ of 100,150 and $250 \mathrm{~mm}$ and exit width $\left(W_{\text {exit }}\right)$ of 20,30 and $40 \mathrm{~mm}$, see Fig. $1 \mathrm{~b}$.

The emulsion is prepared in a large tank $\left(0.5 \mathrm{~m}^{3}\right.$ capacity) equipped with two high shear mixers. The emulsion from the preparation tank is circulated through the pipeline-test rig using a centrifugal pump. The general specifications of the used centrifugal pump and pump performance parameters as manufacturer's data are: Type; N.D25 160/150, Power; 2 hp; Speed; 2900 r.p.m., Head; 20:31 m and Capacity; $7: 15 \mathrm{~m}^{3} / \mathrm{s}$. The temperature during all experiments is maintained constant at $27^{\circ} \mathrm{C}$ with temperature controller. 


\section{Emulsion Preparation}

Two different sets of emulsions are prepared using tap water and a refined white mineral oil. The white mineral oil is low viscosity colorless, tasteless and odorless highly refined paraffinic oil, supplied by CO-OP Company, Alexandria, Egypt. It has a density of $840 \mathrm{~kg} / \mathrm{m}^{3}$ at $27^{\circ} \mathrm{C}$ and a kinematic viscosity limits at $40^{\circ} \mathrm{C}$ from 13 to 19 cSt. In the first set of emulsions, no chemical-emulsifier (surfactant) is added so that unstable emulsion is produced. The unstable emulsion will be separated into oil and water if left without agitation for sometime. The experiments in this set begin with tap water into which a required amount of oil varies from 0.0 to $0.2(20 \%)$ by volume based on the emulsion volume. The holdup $\Phi$ is defined as:

$\Phi=\frac{V_{o}}{V_{o}+V_{w}}=\frac{V_{o}}{V_{E}}$

where, $V_{o}$ is the volume of oil, $V_{w}$ is the volume of water, and $V_{E}$ is the total (emulsion) volume. In the second set of emulsions an ionic surfactant (chemicalemulsifier) namely Sodium Dodecyl Sulfate $\left[\mathrm{CH}_{3}\left(\mathrm{CH}_{2}\right)_{11} \mathrm{OSO}_{3} \mathrm{Na}\right]$ is added to the oil with $1.5 \%$ based on the water weight.

The density and viscosity of pure water and oil-in-water emulsions are measured in laboratories of faculty of sciences, Menoufiya University using densitometer and viscometer. The measuring values of emulsions density and viscosity are summarized in a worksheet table by which the relation between the density as well as viscosity against the holdup for stable and unstable (oil-in-water) emulsions are drawn. Fig. 2 shows the relative density (emulsion density to water density) and relative viscosity (emulsion viscosity to water viscosity) at different holdup values of stable and unstable emulsions at the laboratory temperature $\left(27^{\circ} \mathrm{C}\right)$. As noticed, the density decreases while the viscosity increases as the holdup increases. The present measurements of the discharge coefficient, density and viscosity are considered for the sake of the exact computations of Reynolds numbers at inflow.

\section{Flow Rate Measurements}

The emulsion flow rate is measured by a calibrated orifice meter and controlled by a gate valve placed after the pump. Fig. 3 shows the experimental values of discharge coefficient with different emulsion holdup and different Reynolds number $\rho_{E} D_{p} \bar{V} / \mu_{E}$, where $\rho_{E}$ is the emulsion density, $D_{p}$ is the pipe diameter, $\bar{V}$ is the average emulsion velocity and $\mu_{E}$ is the emulsion viscosity. The calibration curves of the orifice meter are clearly shown in Fig. 3 , in which it is noticed that the discharge coefficient of the orifice meter decreases with the holdup with very small variations with Reynolds number.

\section{Wall Static Pressure Measurements}

Multi-tube manometer has been used to measure the static pressure distributions along the outer and inner walls of the curved diffusers, the upstream pressure and 


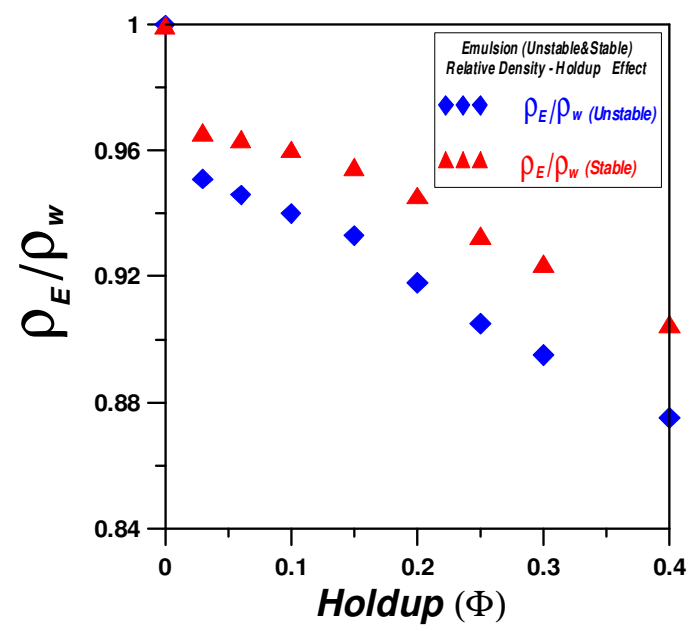

(a)

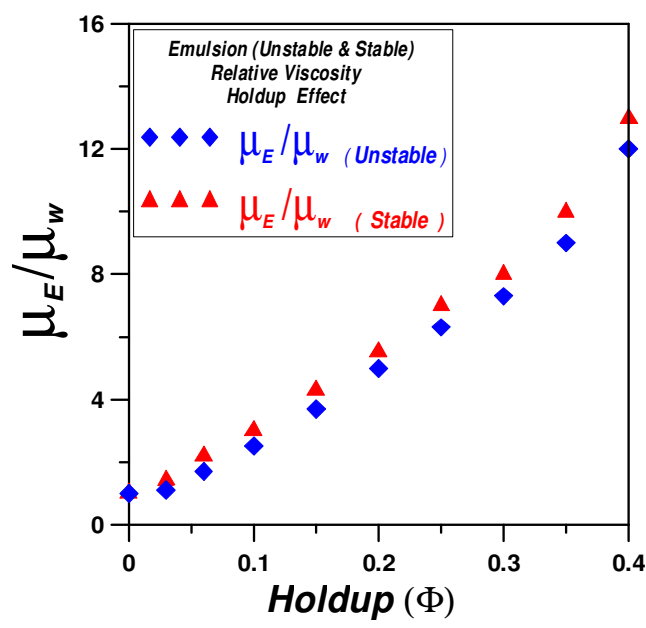

(b)

Fig. 2. Relative density (a) and viscosity (b) of unstable and stable oil-in-water emulsions versus holdup.

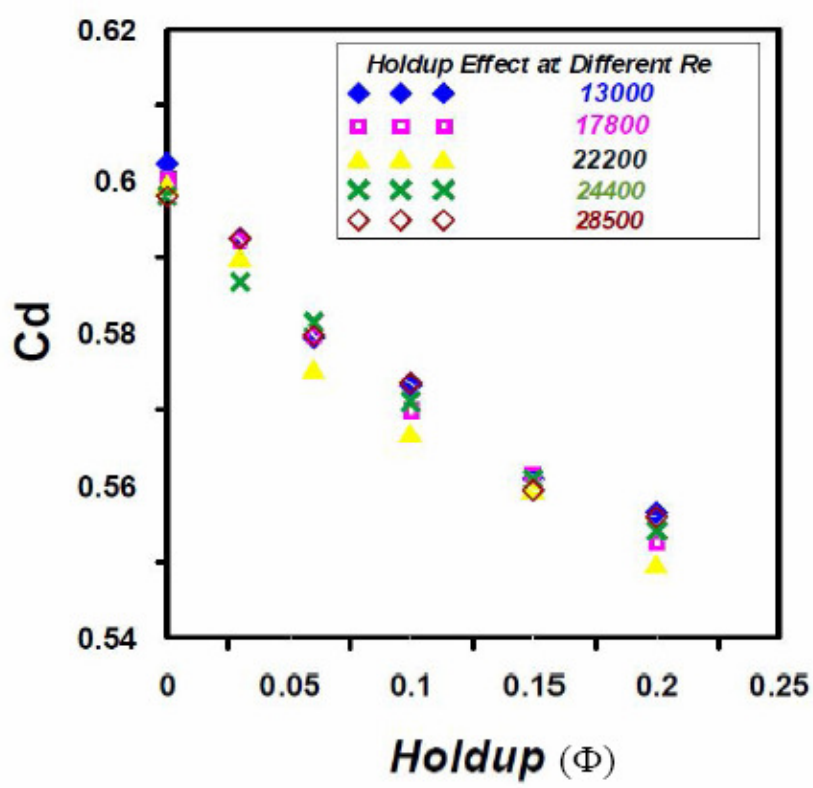

Fig. 3. Discharge coefficient of the orifice meter.

and downstream pressure. The manometer fluid used in the manometer is Carbon Tetra Chloride $\left(C C L_{4}\right)$. This liquid does not mix with water as well as the emulsions and has specific gravity of 1.4 approximately. By using this liquid the static pressure at each tapping hole can be measured by the difference-head of the manometer liquid.

\section{UNCERTAINTY ANALYSIS}

The experimental errors may be of two types, namely fixed and random errors. Fixed error can be removed by proper calibration or correction while random error cannot be avoided. The factors that introduce random error are uncertain by their nature. 
Uncertainty analysis should be conducted on all data collected from all measurements in order to quantify the data and validate the accuracy. As known, the flow resistance coefficient of the tested curved diffuser, $K_{d}$ (curved diffuser-energy loss coefficient) is given by:

$$
K_{d}=h_{\text {loss }} \frac{2 g}{U_{\text {ref }}^{2}}=h_{\text {loss }} \frac{2 g}{\left(Q / A_{\text {inlet }}\right)_{\text {ref }}^{2}}=h_{\text {loss }} \frac{2 g B^{2} W^{2}}{Q^{2}}
$$

In which, $Q\left(\mathrm{~m}^{3} / \mathrm{s}\right)$ is the volume flow rate measured by the calibrated orifice meter and $h_{\text {loss }}$ is the loss head through the curved diffuser. The precision uncertainty of $K_{d}$ is due to uncertainties for all independent variables of them. The precision uncertainty $\delta K_{d} / K_{d}$ can be represented as given by Taylor [15]:

$$
\frac{\delta K_{d}}{K_{d}}= \pm \sqrt{\left[\left(\frac{h_{\text {loss }}}{K_{d}} \frac{\partial K_{d}}{\partial h_{\text {loss }}} \frac{\delta h_{\text {loss }}}{h_{\text {loss }}}\right)^{2}+\left(\frac{B}{K_{d}} \frac{\partial K_{d}}{\partial B} \frac{\delta B}{B}\right)^{2}+\left(\frac{W}{K_{d}} \frac{\partial K_{d}}{\partial W} \frac{\delta W}{W}\right)^{2}+\left(\frac{Q}{K_{d}} \frac{\partial K_{d}}{\partial Q} \frac{\delta Q}{Q}\right)^{2}\right]}
$$

where, $\delta h_{\text {loss }} / h_{\text {loss }}, \delta B / B, \delta W / W$ and $\delta Q / Q$ are the precision uncertainties for the independent variables $h_{\text {loss }}, B, W$ and $Q$, respectively. The reasonable estimate of the uncertainty intervals due to random error in the present experimental study is taken as plus or minus half the smallest scale division (the least count) of the instrument used in conducting the measurement. Thus, $\delta h_{\text {loss }}, \delta B$ and $\delta W$ are taken to be $0.5 \mathrm{~mm}$. The discharge $Q$ is measured using the calibrated orifice meter and calculated from the orifice meter equation as follows:

$$
Q=C_{d} \frac{A_{o} A_{p}}{\sqrt{A_{p}^{2}-A_{o}^{2}}} \sqrt{2 g \Delta H_{o} \frac{\rho_{m}-\rho_{E}}{\rho_{E}}}
$$

where, $C_{d}$ is the orifice discharge coefficient, $A_{o}=\pi d_{o}^{2} / 4$ is the orifice area, $A_{p}=\pi D_{p}^{2} / 4$ is the pipe cross-section area, $\Delta H_{o}$ is the mercury head-difference in the mercury $\mathrm{U}$-tube manometer, $\rho_{m}$ is the mercury density in the manometer and $d_{o}$ is the orifice diameter. Thus,

$$
\frac{\delta Q}{Q}= \pm \sqrt{\left[\left(\frac{\Delta H_{o}}{Q} \frac{\partial Q}{\partial \Delta H_{o}} \frac{\delta \Delta H_{o}}{\Delta H_{o}}\right)^{2}+\left(\frac{D_{p}}{Q} \frac{\partial Q}{\partial D_{p}} \frac{\delta D_{p}}{D_{p}}\right)^{2}+\left(\frac{d_{o}}{Q} \frac{\partial Q}{\partial d_{o}} \frac{\delta d_{o}}{d_{o}}\right)^{2}\right]}
$$

By substituting equation (11) into equation (9), the precision uncertainties of the energy-loss coefficient can be obtained. Table (2) presents the range of energy-loss coefficient $\left(K_{d}\right)$ and percentage precision uncertainty $\left( \pm U_{K_{d}} \%\right)$ of energy-loss coefficient for all measurements. 
Table 2. Uncertainty of the energy loss coefficient for curved diffusers with emulsions.

\begin{tabular}{|c|c|c|c|c|}
\hline No & Model & $\begin{array}{c}\text { Range of } \\
\text { energy loss } \\
\text { coefficient } K_{d}\end{array}$ & $\begin{array}{c}\text { Emulsion } \\
\text { type }\end{array}$ & $\begin{array}{c}\text { The percentage } \\
\text { uncertainty } \\
\pm U_{K_{d}} \%\end{array}$ \\
\hline 1 & Model (1) & $1.9 \leq K_{d} \leq 11$ & Stable & $4 \leq \pm U_{K_{d}} \leq 4.4$ \\
\hline 2 & Model (1) & $1.8 \leq K_{d} \leq 5.4$ & Unstable & $3.1 \leq \pm U_{K_{d}} \leq 3.5$ \\
\hline 3 & Model (2) & $2.4 \leq K_{d} \leq 12$ & Stable & $3.7 \leq \pm U_{K_{d}} \leq 4.2$ \\
\hline 4 & Model (2) & $2.2 \leq K_{d} \leq 7$ & Unstable & $2.9 \leq \pm U_{K_{d}} \leq 3.2$ \\
\hline 5 & Model (3) & $2.9 \leq K_{d} \leq 15$ & Stable & $3 \leq \pm U_{K_{d}} \leq 3.4$ \\
\hline 6 & Model (3) & $2.6 \leq K_{d} \leq 10.75$ & Unstable & $2.4 \leq \pm U_{K_{d}} \leq 2.6$ \\
\hline 7 & Model (5) & $2.7 \leq K_{d} \leq 13.5$ & Stable & $3.4 \leq \pm U_{K_{d}} \leq 3.9$ \\
\hline 8 & Model (5) & $2.3 \leq K_{d} \leq 8.0$ & Unstable & $2.7 \leq \pm U_{K_{d}} \leq 3$ \\
\hline 9 & Model (6) & $3.2 \leq K_{d} \leq 14$ & Stable & $3.2 \leq \pm U_{K_{d}} \leq 3.7$ \\
\hline 10 & Model (6) & $2.4 \leq K_{d} \leq 9.2$ & Unstable & $2.6 \leq \pm U_{K_{d}} \leq 2.9$ \\
\hline
\end{tabular}

\section{RESULTS AND DISCUSSION}

\section{Wall Pressure Distributions}

In the present study, it is aimed to study the energy-loss coefficients (resistance coefficients) for $90^{\circ}$ curved diffusers which have different curvature ratios $\left(C R=R_{c} / W\right): 5,7.5$, and 12.5, and different area ratios: 1, 1.5, and 2. However, the geometrical parameters of all six models of the curved diffusers are given in Table 1. The results are presented for unstable oil-in-water emulsions at different holdup $(\Phi)$ values from 0.03 to 0.06 and for stable oil-in-water emulsions at different holdup values from 0.03 to 0.20 .

The measurements are performed at different Reynolds numbers range of $13,000 \leq \operatorname{Re} \leq 28,500$, where $\operatorname{Re}=\rho_{E} U_{r e f} D_{H} / \mu_{E}$ with $D_{H}=2 W B /(W+B)$ (the hydraulic diameter). The pressure recovery coefficients are first presented to obtain the corresponding energy-loss coefficients of the curved diffusers with pure water as well as oil-in-water emulsion with the previous stated values of holdup for stable and unstable emulsions.

The static pressure recovery coefficient for all diffuser models are presented for pure water in Figs. 4 and 5 at Reynolds number of 28,500. The effects of curvature ratio (Fig. 4) and area ratio (Fig. 5) are clearly shown, respectively. From the experimental results the comparisons in Figs. 4 and 5 reveal that the pure water flow in the curved diffuser model (3) exhibits the lower value in the static pressure recovery coefficient, then model (2) comes in the second stage, and the static pressure recovery coefficient, then model (2) comes in the second stage, and the static pressure recovery coefficient in model (1) comes in the third stage. Also the comparison 


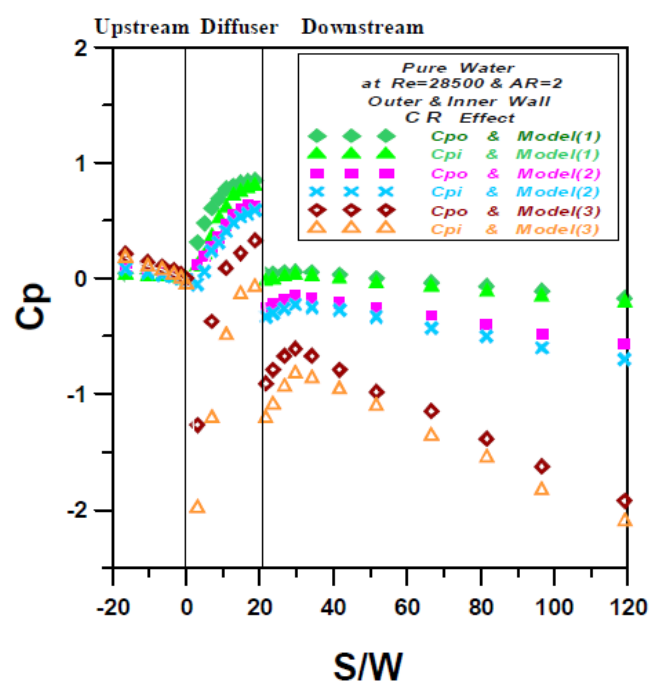

Fig. 4. Effect of curvature ratio on static pressure recovery coefficient on outer and inner walls for models (1), (2) and (3) with

$\mathrm{AR}=2$ and $\mathrm{Re}=28,500$ (water flow).

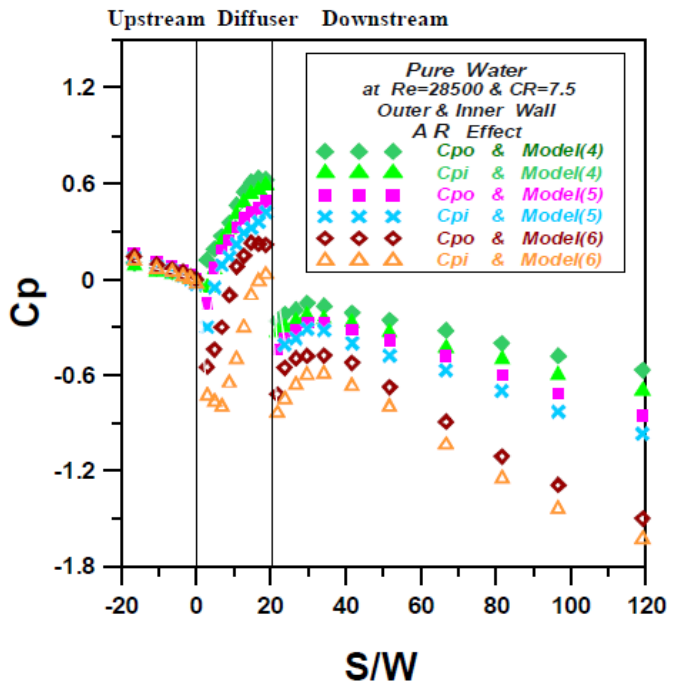

Fig. 5. Effect of area ratio on static pressure recovery coefficient on outer and inner walls for models (4), (5) and (6) with

$\mathrm{CR}=7.5$ and $\mathrm{Re}=28,500$ (water flow).

shows that the static pressure recovery coefficient of the curved diffusers with pure water increases as the area ratio or curvature ratio increases. This behavior tends to lower the resistance to flow in case of high area ratio or curvature ratio. The same behavior is noticed with increasing the area ratio (models 4, 5 and 6) as shown in Fig. 5. Generally, the higher wall pressure distribution is observed on the outer walls of all models, because of the presence of radial pressure gradient generated by the centrifugal force.

The static pressure recovery coefficients for all models are also presented for $\Phi=0.15$ stable oil-in-water emulsions in Figs. 6 and 7 on the outer and inner walls at Reynolds number of 28,500. By comparing the results in Figs. 6 and 7, it is found that the static pressure recovery coefficient increases as the area ratio or curvature ratio increases. This because as the area ratio, $(A R)$ increases from 1.0 to 2.0, the emulsion flow resistance decreases and also the increase of curvature ratio from 5.0 to 12.5 leads to the emulsion flow resistance decreases. Comparing the presence of 0.15 oil concentration with case of water only, one notices that there is a distinct reduction of pressure along the walls of curved diffuser (compare Fig. 4 with Fig. 6 and Fig. 5 with Fig. 7).

The static pressure recovery coefficient for model (5) is presented for a stable emulsion of 0.20 oil concentration in Fig. 8 along the outer and inner walls at different emulsion flow Reynolds numbers, ranged from 13,000 to 28,500 . It is noticed that the wall-pressure recovery coefficient of the curved diffusers with 0.20 stable oil-in-water emulsions is directly proportional to the flow Reynolds number with a noticeable decrease in the adverse pressure gradient inside the diffuser. This is due to the decrease of boundary layer thickness on the walls and hence reduces the chances of separation in the curved diffuser. Therefore, the performance of curved diffuser can be improved with accelerating the inflow. 


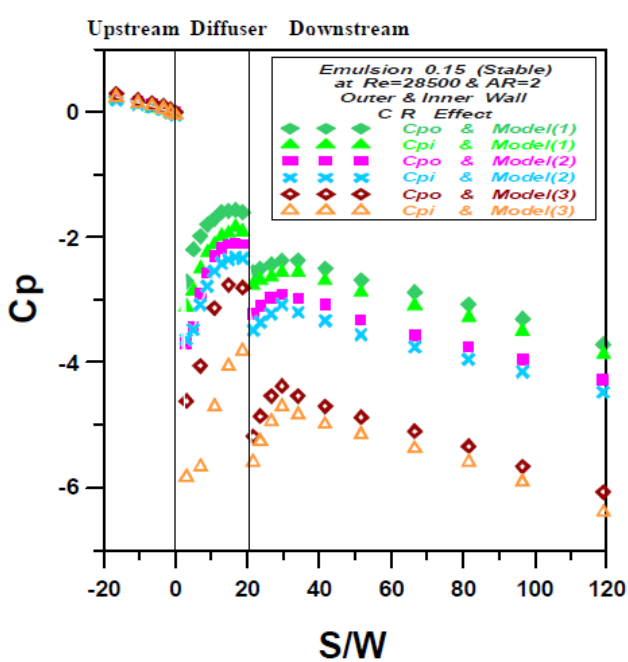

Fig. 6. Effect of curvature ratio on static pressure recovery coefficient on outer and inner walls for models (1), (2) and (3) with $A R=2$ and $R e=28,500$ (stable emulsion flow with $\Phi=0.15$ ).

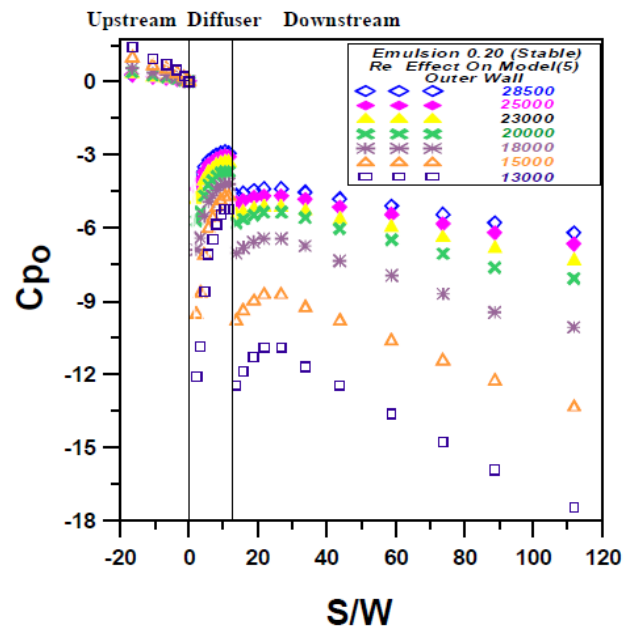

(a)

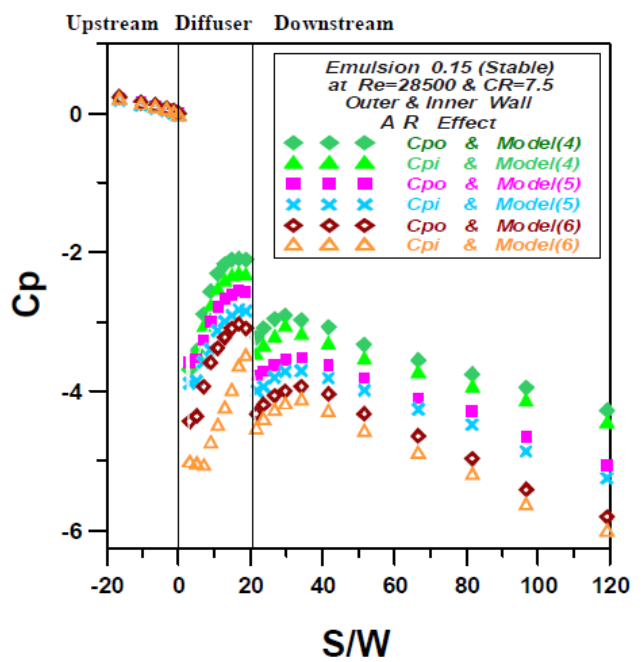

Fig. 7. Effect of area ratio on static pressure recovery coefficient on outer and inner walls for models (4), (5) and (6) with $\mathrm{CR}=7.5$ and $\mathrm{Re}=28,500$ (stable emulsion flow with $\Phi=0.15$ ).

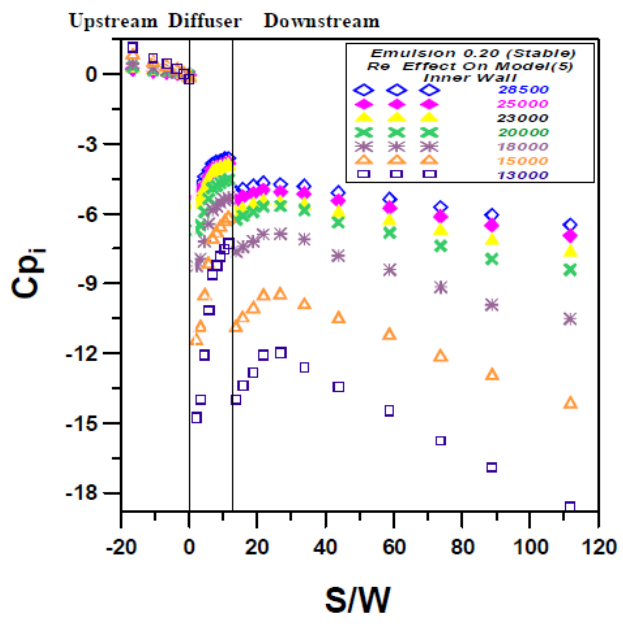

(b)

Fig. 8. Effect of Reynolds number on static pressure recovery coefficient on outer wall (a) and inner wall (b) for model (5) with $\mathrm{CR}=7.5$ (stable emulsion flow with $\Phi=0.2$ ).

The effects of holdup on the wall-pressure recovery coefficients of model (1) are significant. This is clearly visible for pure water and stable oil-in-water emulsions in Fig. 9 on the outer and inner walls of model (1). The emulsion holdup values range from 0.03 to 0.20 and with inflow Reynolds number of 28,500. It is clear that the static pressure recovery coefficient of the curved diffusers decreases as the dispersed phase holdup increases, creating a strong adverse pressure gradient inside the curved diffuser. The presence of oil increases the frictional drag of water to move in the curved diffuser and this enhances the energy loss of the curved diffuser. 


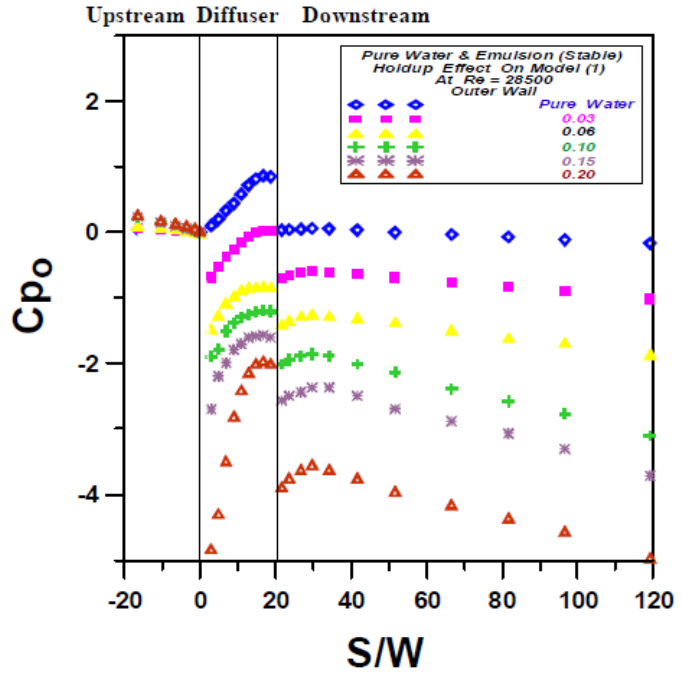

(a)

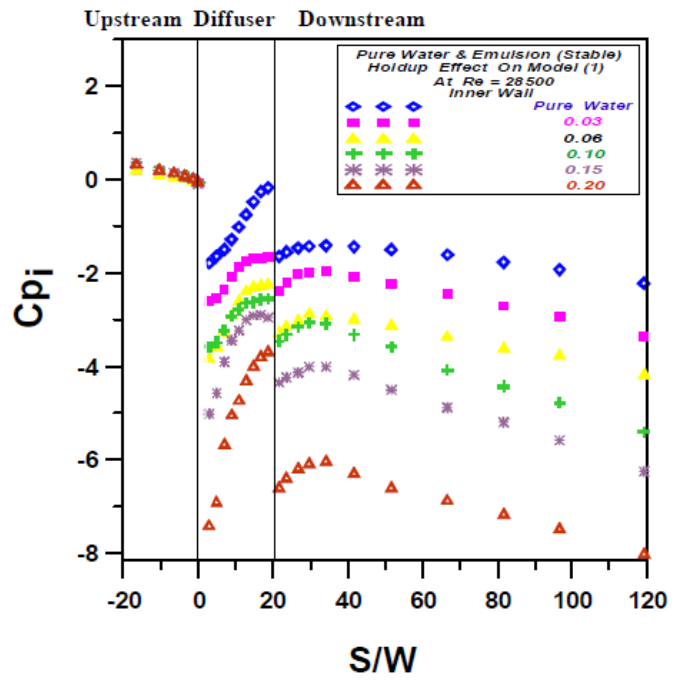

(b)

Fig. 9. Effect of holdup $(\Phi)$ on static pressure recovery coefficient on outer wall (a) and inner wall (b) for model (1) with $\mathrm{CR}=12.5$ and $\mathrm{Re}=28,500$ (water and stable emulsion flows with $0.03 \leq \Phi \leq 0.20$ ).

\section{Diffuser Performance}

In the present section, attempts have been made to understand the performance of emulsion flow in curved diffuser in terms of the energy-loss characteristics. The diffuser energy-loss coefficient is expressed in terms of inflow Reynolds number. The effects of holdup, area ratio, and curvature ratio will be discussed.

The energy loss coefficients of all tested curved diffuser models are presented for pure water as well as stable emulsion in Fig. 10. Emulsion results are considered at different holdup values ranges from 0.03 to 0.20 . From the experimental results, the energy-loss coefficient of the curved diffusers is found to be directly proportional to the dispersed phase holdup, which is in agreement with Khalil et al [9]. Generally, the energy loss coefficient decreases with Reynolds number because of the reduction of adverse pressure gradient generated in the curved diffuser, while it increases with the emulsion holdup because of the increased drag due to the presence of oil in water which is responsible of the emulsion-viscosity increase.

The effects of surfactant presence (stable emulsion) or absence (unstable emulsion) in emulsion on the energy loss in curved diffusers are presented in Figs. 11 and 12. The study includes the variation of curvature ratio (Fig. 11) and the variation of area ratio (Fig. 12) at a constant holdup of 0.06 . At the same Reynolds number, the energy-loss coefficient of the curved diffuser decreases with increasing either the curvature ratio or area ratio. The main reason of the curvature effects is the decreasing of the centrifugal force created in the curved diffuser with increasing the curvature ratio, which is responsible for secondary flow generation. For the same radius of curvature, i.e. an equal main-flow passage, the pressure gain from the widest diffuser (the highest area ratio) is higher than the energy lost due intensive secondary flows produced. It means that the energy loss decreases with increasing the area ratio. The stable emulsion exhibits greater values of the curved-diffuser 


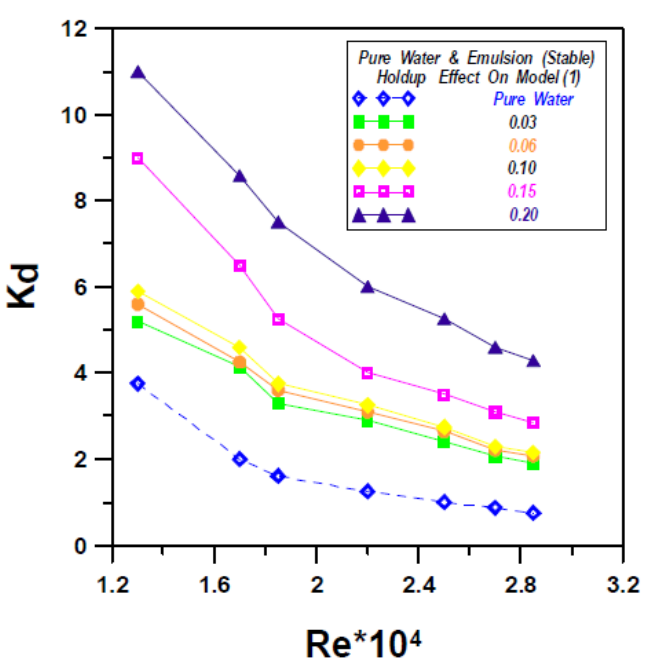

(a) Model (1)

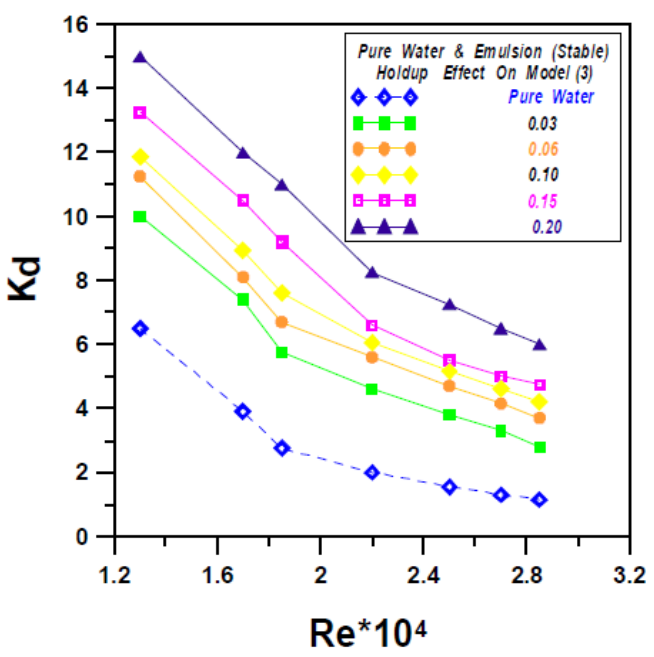

(c) Model (3)

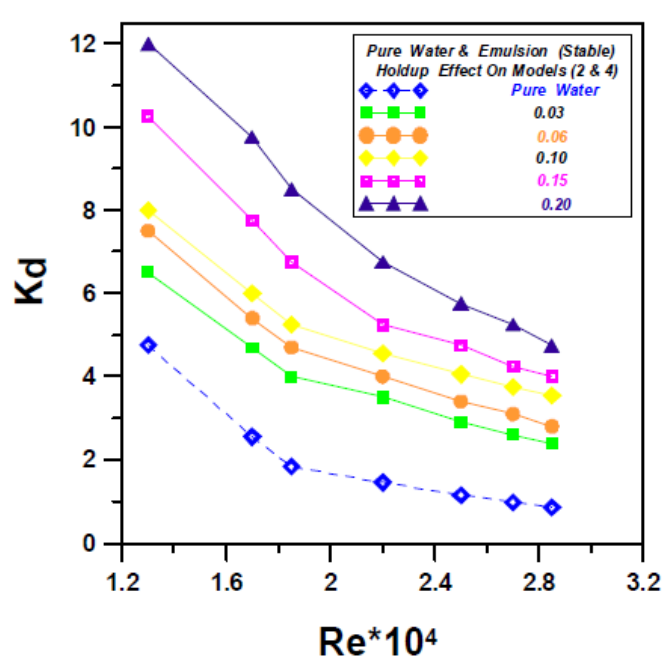

(b) Models ( 2 \& 4)

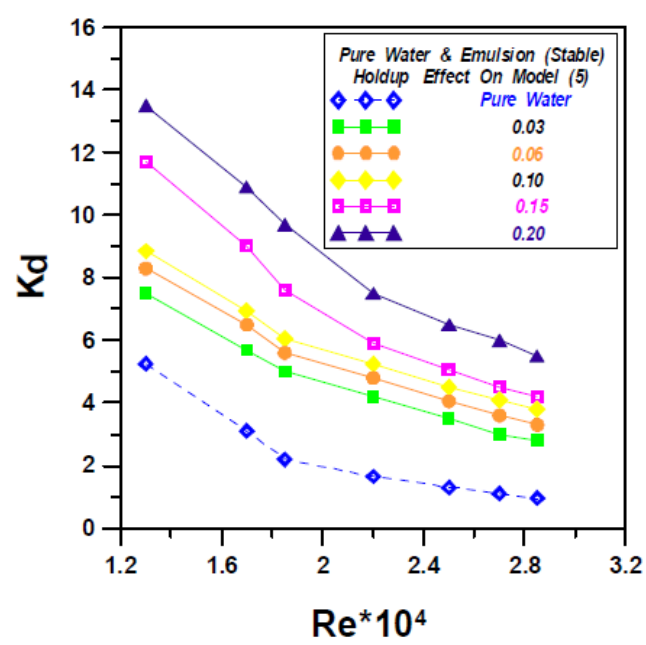

(d) Model (5)

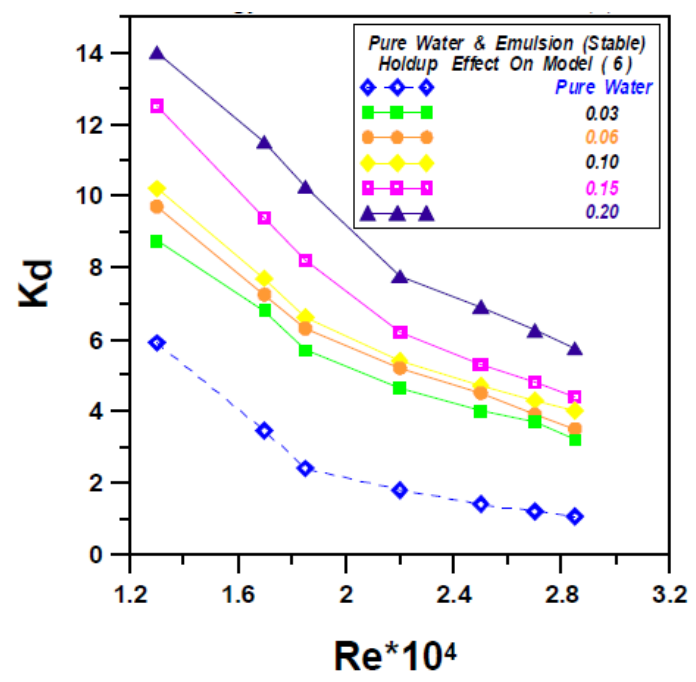

(e) Model (6)

Fig. 10. Diffuser-loss coefficient against Reynolds number (water and stable emulsion flows with $0.03 \leq \Phi \leq 0.20$ ). 


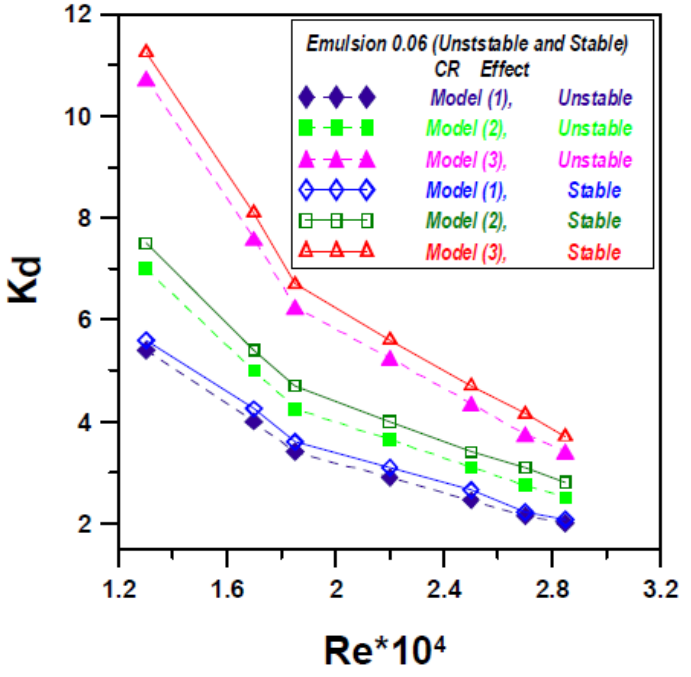

Fig. 11. Effect of curvature ratio on diffuser-loss coefficient (water, stable and unstable emulsion flows with $\Phi=0.06$ ).

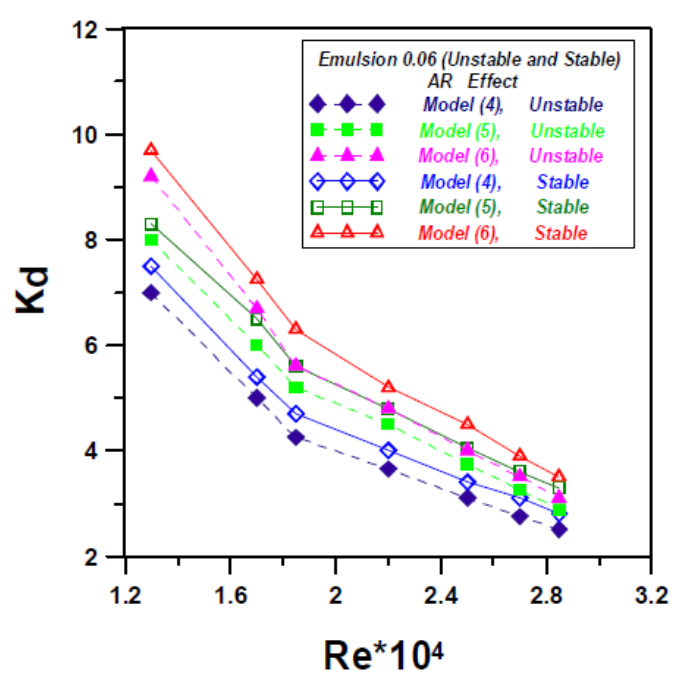

Fig. 12. Effect of area ratio on diffuserloss coefficient (water, stable and unstable emulsion flows with $\Phi=0.06$ ).

energy-loss coefficient than the unstable emulsion $\left[K_{d}\right.$ (stableemulsion) $>K_{d}$ (unstable-emulsion)]. This behavior is attributed to the change of turbulence intensity which may be affected by the dispersed phase holdup, area ratio and curvature ratio of the diffuser.

Fig. 13 shows curvature-ratio effects on the energy-loss coefficients of the curved diffuser. The results of water and unstable emulsions flows with two different holdup values ( $\Phi=0.03$ and 0.06 ) are represented in the figure. As discussed previously, as the curvature ratio decreases the energy loss in the curved diffuser increases for the different flows, because of the generation of strong secondary flows (with small radius of curvature) superimposed on the mean flow.

The curvature ratio effects are again for water and 0.15 holdup stable oil-in-water emulsions in Fig. 14. It is revealed that the pure water flow in curved diffusers exhibits the lower values in the energy loss coefficient compared with the stable emulsion flow. Also the created secondary flows because the strong turn of curved diffuser with small curvature radius clearly increase the curved-diffuser loss coefficient. Generally, the energy-loss coefficient of the curved diffusers for pure water and stable and unstable oil-in-water emulsions is found to be inversely proportional to the curvature ratio, area ratio of the curved diffusers and the flow Reynolds number and to approach asymptotic values for high flow Reynolds number due to the intensive turbulence intensity.

However, a general correlation of the loss coefficient (not previously considered) for the present cases exploring the ranges of geometrical parameters, inflow condition 


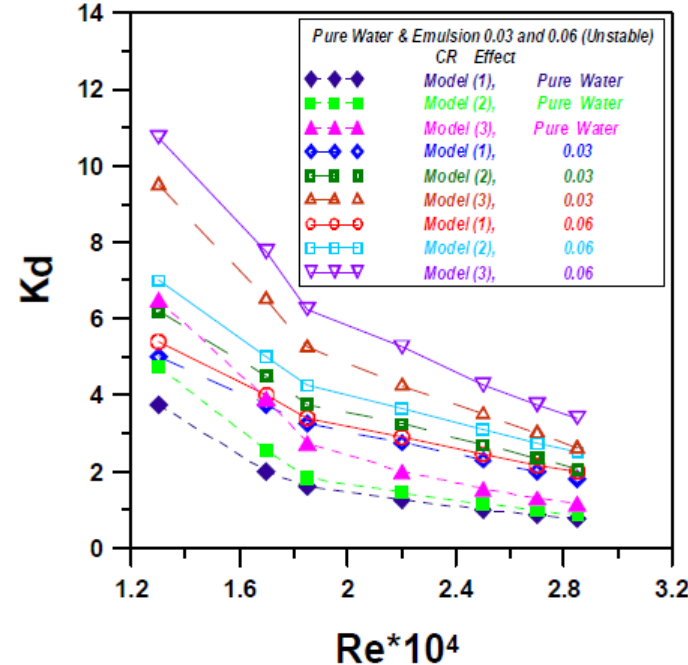

Fig. 13. Effect of curvature ratio and holdup on diffuser-loss coefficient (water and unstable emulsion flows with $\Phi=0.03,0.06$ ).

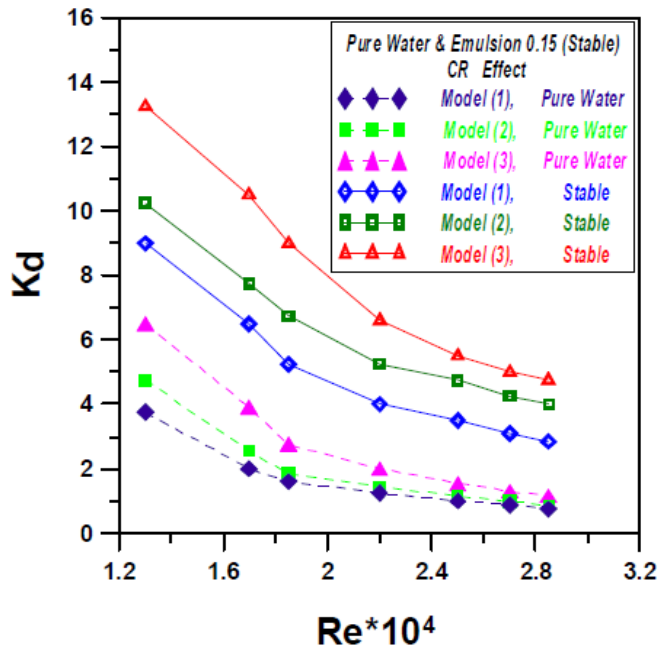

Fig. 14. Effect of curvature ratio on diffuser-loss coefficient (water and stable emulsion flows with $\Phi=0.15$ ).

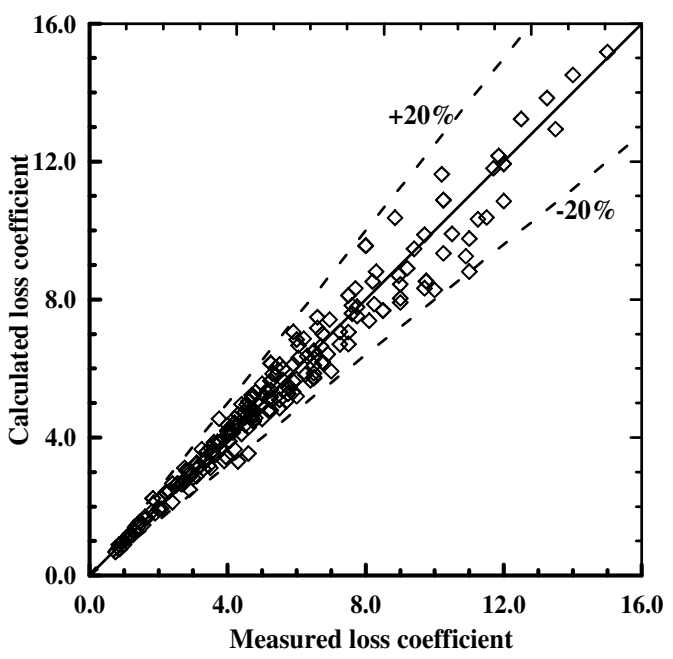

Fig. 15. Calculated loss coefficient against the measured loss coefficient.

and Reynolds number conditions can be extracted from the present experimental study. The correlation reads:

$K_{d}=\frac{\alpha \Phi^{\beta}}{C R^{\gamma} A R^{\omega} \mathrm{Re}^{\zeta}}$

The corresponding correlation coefficients are tabulated in Table 3 for pure water as well as the emulsion cases. Fig. 15 represents the correlated loss coefficient against the measured one showing a maximum error range of $\pm 20 \%$. 
Table 3. Correlation coefficients of the loss coefficient.

\begin{tabular}{|c|c|c|c|c|c|}
\hline Flow & $\alpha$ & $\beta$ & $\gamma$ & $\omega$ & $\zeta$ \\
\hline Water & $86 \times 10^{8}$ & 0 & 0.536 & 0.2549 & 2.11853 \\
\hline Emulsion & $19.778 \times 10^{5}$ & 0.3197 & 0.5936 & 0.2827 & 1.2493 \\
\hline
\end{tabular}

\section{CONCLUSIONS}

Based on the experimental results, and related discussion the following conclusions can be drawn:

1- The $\mathrm{o} / \mathrm{w}$ emulsion density increases as the holdup decreases, but the $\mathrm{o} / \mathrm{w}$ emulsion viscosity increases as the holdup increases.

2- For all tested models the pressure recovery coefficient $\left(C_{p}\right)$ decreases through the upstream tangent and the downstream tangent by different values from a curved diffuser model to other. However, it increases through the curved diffuser by different values from one model to other.

3- Under the present range of Reynolds number condition (13000 - 28500), the curved diffusers pressure recovery coefficients with the stable and unstable $\mathrm{o} / \mathrm{w}$ emulsions are lower than that for pure water. The unstable o/w emulsion exhibits the highest values compared with stable o/w emulsion.

4- The pressure recovery coefficient decreases as the holdup increases and inflow Reynolds number decreases.

5- Generally, for all tested models the outer wall pressure recovery coefficient is higher than that the inner wall.

6- The curved diffuser energy-loss coefficients with stable and unstable o/w emulsions are higher than that with pure water.

7- The unstable oil-in-water emulsion exhibits lower values in energy-loss coefficients, compared with stable oil-in-water emulsion.

8- The energy-loss coefficient is found to be inversely proportional to the Reynolds number, which is in agreement with that given in literature.

9- For curved diffusers as the area ratio (AR) increases from 1.0 to 2.0, the energyloss coefficient decreases and as the curvature ratio $\left(R_{c} / W\right)$ increases from 5 to 12.5 , the energy-loss coefficient decreases.

Finally, the results and conclusions given in this paper will be useful for those who are working in chemical and petroleum industries. In order to extract a general conclusion, it is recommended to consider wide ranges of holdup, Reynolds number, curvature ratio and area ratio. This will be considered in future work. 


\section{REFERENCES}

[1] Pal, R., Rhodes, E., Emulsion Flow in Pipelines, Int. J. Multiphase Flow Vol. 15, No. 6, pp. 1011-1017, (1989).

[2] Pal, R., Pipeline Flow of Unstable and Surfactant Stabilized Emulsions, AICHE Journal, Vol. 39, No. 11, pp. 1754-1764, (1993).

[3] Hwang, C-Yi J., Pal, R., Flow of Two-Phase Oil/Water Mixture through Sudden Expansions and Contractions, Chemical Engineering J. Vol. 68 (Issues 2-3), pp. 157-163, (1997).

[4] Turian, R.M., Ma, T.-W., Hsu, F.-L.G., Sung, D.-J., Plackmann, G.W., Flow of Concentrated Non-Newtonian Slurries: 2. Friction Losses in Bends, Fittings, Valves, and Venturi Meters, Int. J. Multiphase Flow, Vol. 24, pp. 243-269, (1998).

[5] Pal, R., Hwang, C-Yi J., Loss Coefficients for Flow of Surfactant-Stabilised Emulsions through Pipe Components, Trans. IChemE Part A 77, pp. 685691, (1999).

[6] Khalil, M.F., Kassab, S.Z., Elhalawany, M.M., Effect of Surfactant Additives on Energy Loss in Pipe Fittings, $12^{\text {th }}$ International Mechanical Power Engineering Conference, Mansora, Egypt, (IMPEC 12), F143-F159, (2001).

[7] Langevin, D., Poteau, S., Henaut, I., Argillier, J.F., Crude Oil Emulsion Properties and their Application to Heavy Oil Transportation, Oil \& Gas Science and Technology-Rev. IFP, Vol. 59, No. 5, pp. 511-521, (2004).

[8] Ismail, A.S., Modeling Friction Factor and Drag Reduction of Unstable Waterin-Oil Emulsions in Horizontal Pipes, Al-Azhar University Engineering Journal, Vol. 8, pp. 10-24, (2005).

[9] Khalil, M.F., Kassab, S.Z., Ismail, A.S. Elazab, I.S., Friction Losses of Oil-inWater Emulsions Flow through Pipes, Cairo 10th International Conference on Energy and Enviroment (EE10), Luxor, Egypt, (2007).

[10] Fester, V., Mbiya, B., Slatter, P., Energy Losses of Non-Newtonian Fluids in Sudden Pipe Contractions, Chemical Engineering J. Vol. 145, No. 1, pp. 5763, (2008).

[11] El-Askary, W.A., Nasr, M., Performance of a Bend-Diffuser System: Experimental and Numerical Studies, J. of Computers and Fluids Vol. 38, No. 1, pp. 160-170, (2009).

[12] Balakhrisna, T., Ghosh, S., Das, P.K., Oil-Water Flows through Sudden Contraction and Expansion in a Horizontal Pipe, Phase-Distribution and Pressure Drop, Int. J. Multiphase Flow Vol. 36, No. 1, pp. 13-24, (2010).

[13] Hammoud, A.H., Yassine, K.C., Khalil, M.F., Effect of Oil-in-Water Concentration on the Performance of Centrifugal Pump, 10th International Congress of Fluid Dynamics (ICFD 10), Ain Soukhna, Red Sea, Egypt, Paper No. ICFD10-EG-3067, (2010).

[14] Khalil, M.F., Kassab, S.Z., Ismail, A.S., Elazab, I.S., Energy Losses of Oil-inWater Emulsions Flow through Pipe Fittings Using Image Processing, Int. Review of Mechanical Engineering (I. RE.M.E.), (2011).

[15] Taylor, J.R., An Introduction to Error Analysis. University Science Books, Mill Valley, California, (1982). 